\title{
XMM-Newton observations of the INTEGRAL X-ray transient IGR J17544-2619
}

\author{
R. González-Riestra ${ }^{1}$, T. Oosterbroek ${ }^{2}$, E. Kuulkers² ${ }^{2}$ A. Orr² ${ }^{2}$ and A. N. Parmar ${ }^{3}$ \\ 1 XMM-Newton Science Operation Centre, ESA, VILSPA, PO Box 50727, 28080 Madrid, Spain \\ 2 INTEGRAL Science Operations Centre, Science Operations and Data Systems Division, \\ Research and Scientific Support Department of ESA, ESTEC, Postbus 299, 2200 AG Noordwijk, The Netherlands \\ 3 Astrophysics Missions Division, Research and Scientific Support Department of ESA, ESTEC, Postbus 299, \\ 2200 AG Noordwijk, The Netherlands
}

Received 17 November 2003 / Accepted 12 February 2004

\begin{abstract}
On September 17, 2003 INTEGRAL discovered a bright transient source $3^{\circ}$ from the Galactic Center, IGR J17544-2619. The field containing the transient was observed by XMM-Newton on March 17 and September 11 and 17, 2003. A bright source, at a position consistent with the INTEGRAL location, was detected by the European Photon Imaging Camera (EPIC) during both September observations with mean $0.5-10 \mathrm{keV}$ unabsorbed luminosities of $1.1 \times 10^{35}$ and $5.7 \times 10^{35} \mathrm{erg} \mathrm{s}^{-1}$ for an (assumed) distance of $8 \mathrm{kpc}$. The source was not detected in March 2003, with a $0.5-10 \mathrm{keV} \mathrm{lu}-$ minosity of $<3.8 \times 10^{32} \mathrm{erg} \mathrm{s}^{-1}$. The September 11 and 17 EPIC spectra can be represented by a power-law model with photon indices of $2.25 \pm 0.15$ and $1.42 \pm 0.09$, respectively. Thus, the $0.5-10 \mathrm{keV}$ spectrum hardens with increasing intensity. The low-energy absorption during both September observations is comparable to the interstellar value. The X-ray lightcurves for both September observations show energy dependent flaring which may be modeled by changes in either low-energy absorption or power-law index.
\end{abstract}

Key words. accretion, accretion disks - X-rays: individuals: IGR J17544-2619 - stars: neutron - X-rays: binaries

\section{Introduction}

About a dozen new hard X-ray transients have been discovered in the last year during INTEGRAL (Winkler et al. 2003) observations of the galactic center region with the soft gamma-ray imager IBIS/ISGRI (Lebrun et al. 2003). Their unusual spectral hardness has led to suggestions that these sources comprise a group of highly absorbed galactic binaries (Revnivtsev et al. 2003). These are being preferentially detected due to the good sensitivity and large field of view of IBIS/ISGRI above $15 \mathrm{keV}$. XMM-Newton observations of the first of these, IGR J16318-4848, revealed intense $\mathrm{Fe} \mathrm{K} \alpha$ and $\mathrm{K} \beta$ and $\mathrm{Ni} \mathrm{K} \alpha$ emission lines as well as strong low-energy absorption (Matt \& Guainazzi 2003; Walter et al. 2003). XMM-Newton observations of IGR J16320-4851 revealed a featureless hard continuum (Rodriguez et al. 2003). IGR 16358-4726 was observed by Chandra and showed a hard power-law spectrum with a $5880 \mathrm{~s}$ periodic intensity modulation (Patel et al. 2003).

We report on a new bright transient source, IGR J17544-2619, discovered using IBIS/ISGRI on September 17, 2003 at 01:10 UTC during an observation

Send offprint requests to: R. González-Riestra, e-mail: rgonzale@xmm.vilspa.esa.es of the Galactic Center region (Sunyaev et al. 2003). The source intensity was about $160 \mathrm{mCrab}, 60 \mathrm{mCrab}$, and $<15 \mathrm{mCrab}$ (at $3 \sigma$ confidence) in the $18-25 \mathrm{keV}, 25-50 \mathrm{keV}$ and 50-100 keV energy ranges. IGR J17544-2619 was bright for around $2 \mathrm{~h}$ and then faded below the IBIS/ISGRI detection threshold. The source was again detected in outburst by IBIS/ISGRI later the same day between 6 and $14 \mathrm{~h}$ UTC (Grebenev et al. 2003).

By chance, XMM-Newton observed the region of sky containing IGR J17544-2619 only 5 days before the INTEGRAL discovery. A source was clearly detected at a position consistent with that reported from INTEGRAL data, with a mean $2-10 \mathrm{keV}$ intensity of $4.5 \times 10^{-12} \mathrm{erg} \mathrm{cm}^{-2} \mathrm{~s}^{-1}$ (González-Riestra et al. 2003). Rodriguez (2003) reported a possible optical/infrared counterpart in the USNO B1.0 cata$\log (B=13.9-14.5 \pm 0.3 \mathrm{mag})$ and 2MASS all-sky quick-look image archive. He also noted that there are 3 fainter candidates within the preliminary $10^{\prime \prime}$ XMM-Newton uncertainty region in the 2MASS image.

The field containing IGR J17544-2619 was observed three times by XMM-Newton. The first two observations (March 7 and September 11, 2003) were part of a program to study the nova V4643 Sgr (see also González-Riestra et al. 2003), and the 
Table 1. XMM-Newton observation log. The EPIC modes are Full Frame (FF) and Timing (TI). In the March 17, 2003 observation IGR J17544-2619 was outside the OM field of view. The effective wavelengths of the UVW1, UVW2 and UVM2 filters are $2945 \AA$, $2180 \AA$, and $2340 \AA$, respectively.

\begin{tabular}{lccccccccc}
\hline \hline Obs. & Start time & End time & \multicolumn{2}{c}{ Exposure (ks) } & \multicolumn{3}{c}{ EPIC mode } & \multicolumn{3}{c}{ OM } \\
& (UTC) & (UTC) & pn & MOS & pn & MOS1 & MOS2 & Exp (ks) & Filter \\
\hline 1 & 2003 Mar. 17 20:53 & Mar. 18 00:33 & 10.2 & 11.9 & FF & FF & FF & $\ldots$ & $\ldots$ \\
2 & 2003 Sep. 11 17:54 & Sep. 11 22:09 & 9.3 & 11.0 & FF & FF & FF & $2.2,3.2$ & $B, U V W 1$ \\
3 & 2003 Sep. 17 17:13 & Sep. 17 19:59 & 2.5 & 8.3 & TI & TI & FF & $1.8,1.6$ & $U V M 2, U V W 2$ \\
\hline
\end{tabular}

third was a Target of Opportunity observation triggered by the INTEGRAL discovery. Here, we present results from all three XMM-Newton observations.

The XMM-Newton Observatory (Jansen et al. 2001) includes three $1500 \mathrm{~cm}^{2} \mathrm{X}$-ray telescopes each with a European Photon Imaging Camera (EPIC) at the focus. Reflection Grating Spectrometers (RGS, den Herder et al. 2001) are located behind two of the telescopes. In addition, a coaligned optical/UV Monitor (OM, Mason et al. 2001) is included. Two of the EPIC imaging spectrometers use MOS CCDs (Turner et al. 2001) and one uses a pn CCD (Strüder et al. 2001).

\section{Observations and data reduction}

Table 1 gives observing times, exposures and instrument modes. In the first two observations the three EPIC detectors were operated in the Full Frame mode, while during the third observation the pn and the MOS1 were operated in Timing Mode. In this mode, data are collapsed into a one dimensional row to be read at high speed with the second dimension being replaced by timing information. This allows time resolutions of $1.5 \mathrm{~ms}$ and $0.03 \mathrm{~ms}$ for the MOS and pn, respectively. Except for the March 2003 MOS2 observation, performed with the medium filter, the EPIC thin filters were used throughout.

EPIC data were processed with the standard XMM-Newton SAS tasks "epchain" and "emchain". For the pn and MOS imaging data, source counts were extracted from circular regions of $40^{\prime \prime}$ radius centered on IGR J17544-2619. Background counts were obtained from a similar region offset from the source position for pn, and from an annulus around the source for MOS2. Spectra were extracted separately for single, double and single+double events. For timing data, the source spectra were extracted from columns centered on the source position, and the background spectra from offset columns at both sides of the source.

The XMM-Newton OM (Optical Monitor) was operated in imaging mode during the three observations (see also Table 1). During the March 2003 observation IGR J17544-2619 was outside the OM field of view. The OM data were processed with the standard SAS task "omichain".

\section{Results}

\subsection{Source position}

Unfortunately, during the September 11, 2003 observation IGR J17544-2619 was in one of the gaps between the
MOS CCDs. In the September 17 observation the MOS2 data suffer slightly from the effects of pile-up. The pn data of September 11, 2003, therefore, provide the best estimate of the $(\mathrm{J} 2000)$ source position of $\mathrm{RA}=17^{\mathrm{h}} 54^{\mathrm{m}} 25^{\mathrm{s}} .37 \mathrm{Dec}=$ $-26^{\circ} 19^{\prime} 52^{\prime \prime}$, 9 , with a $90 \%$ confidence uncertainty radius of $4^{\prime \prime}$. This position improves on that presented in Gonzalez-Riestra et al. (2003), which was based on a preliminary estimate of the spacecraft attitude and clearly excludes 1RXS J175428.3-2620 (Wijnands 2003). Figure 1 shows the EPIC-pn and OM $B$ filter images with the 2 ' INTEGRAL uncertainty region of Sunyaev et al. (2003) superposed.

Examination of the September $11 \mathrm{OM}$ data reveals that there is a source close to the edge of the refined EPIC pn uncertainty region (see Fig. 1) with a $B$ magnitude of $14.57 \pm$ 0.02 . This is almost certainly the USNO B1.0 catalog source (2MASS J17542527-2619526) discussed in Rodriguez (2003). The OM source is $3^{\prime \prime}$ from the EPIC pn best source position. There is a similar offset $\left(4^{\prime \prime}\right)$ between the OM and the EPIC pn coordinates of V4643 Sgr. Once a correction for this offset is made, the difference between the EPIC pn coordinates and those of the optical star inside the error circle is only 1 .' 2 . The same target is present in the ultraviolet image with a $U V W 1$ magnitude of $16.13 \pm 0.04$, and in the $U V W 2$ image of September 17 with $U V W 2=14.49 \pm 0.02$. The source is not detected in the $U V M 2$ image.

In the March 2003 observation only X-ray upper limits to the intensity of a source at the position of IGR J17544-2619 are obtained. The $0.5-10 \mathrm{keV}$ pn $3 \sigma$ upper limit of 0.008 count s$^{-1}$ corresponds to a flux of $5 \times 10^{-14} \mathrm{erg} \mathrm{cm}^{-2} \mathrm{~s}^{-1}$ for a powerlaw spectrum with a photon index, $\alpha$, of 2.0 and an $N_{\mathrm{H}}$ of $1.4 \times 10^{22}$ atom $\mathrm{cm}^{-2}$ (the interstellar value in the direction of IGR J17544-2619; Dickey \& Lockman 1990).

\subsection{X-ray light curves}

The $0.5-10 \mathrm{keV}$ IGR J17544-2619 light curves and hardness ratios (counts in the energy range $2-10 \mathrm{keV}$ divided by those between $0.5-2 \mathrm{keV}$ ) from both September observations are shown in Fig. 2. Since for the September 17 observation pn data are available only after 19:15 UTC due to technical problems, we show here the MOS1 data. In order to compare the two light curves, it is worth noting that during this overlapping interval 1 MOS 1 count s ${ }^{-1}$ corresponds to $\sim 3.5$ pn count $\mathrm{s}^{-1}$.

During the September 11 observation the $0.5-10 \mathrm{keV}$ pn count rate increased from $0.06 \mathrm{~s}^{-1}$ to $\sim 3 \mathrm{~s}^{-1}$ in about $500 \mathrm{~s}$. A short ( 100s) flare occurred at 20:25 UTC when the count 


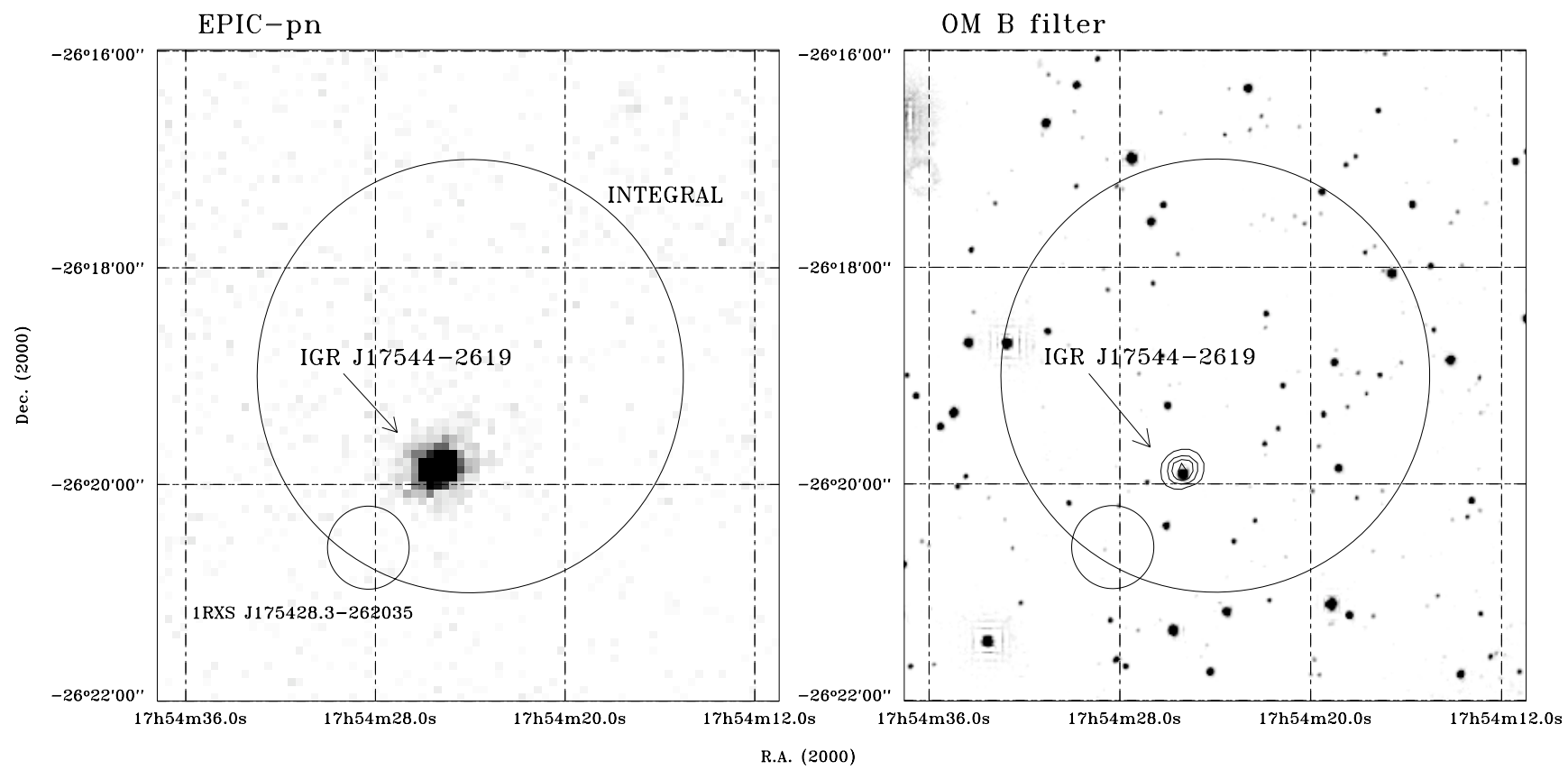

Fig. 1. 2003 September 11 EPIC-pn (left) and OM B filter (right) images of the area around the $2^{\prime}$ INTEGRAL uncertainty region for IGR J17544-2619 of Sunyaev et al. (2003, big circle). The contours shown in the optical image correspond to the EPIC-pn image (0.005, $0.010,0.015,0.020$ and 0.025 counts $\mathrm{s}^{-1}$ ), which clearly excludes 1RXS J175428.3-262035 (small circle).
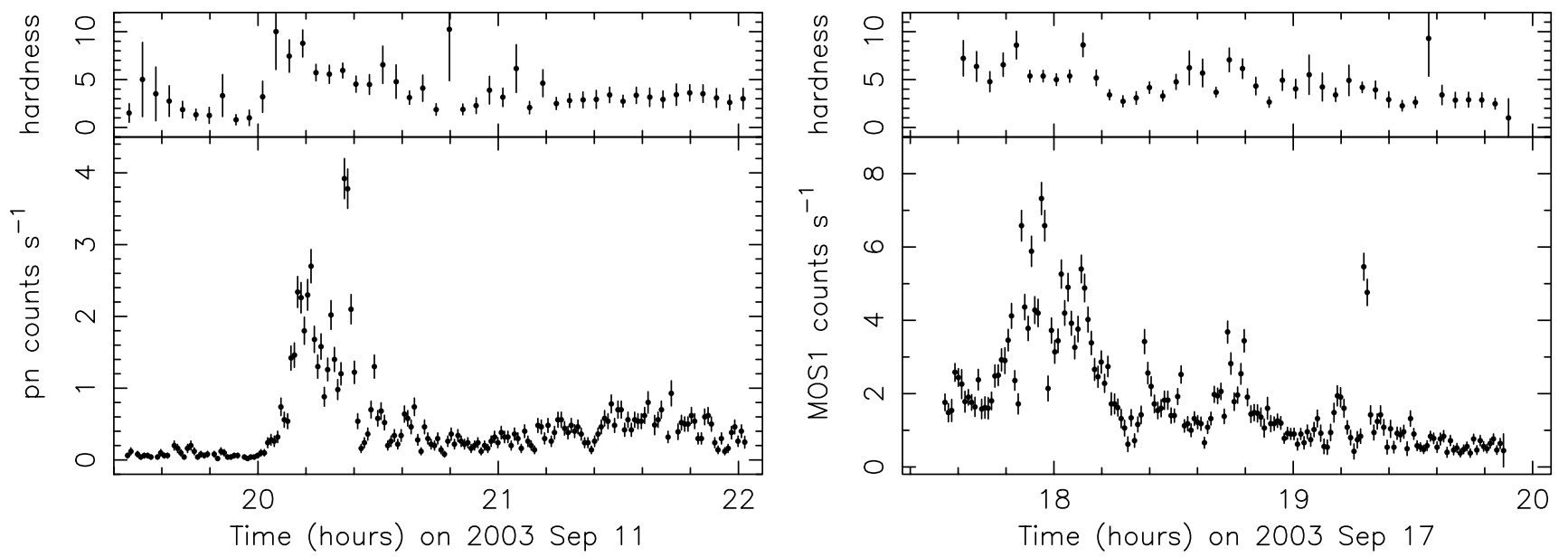

Fig. 2. IGR J17544-2619 0.5-10 keV light curves for the September 11 (pn; lower left) and September 17, 2003 (MOS1; lower right) observations with integration times of $50 \mathrm{~s}$. A MOS 1 count rate of 1 count s$^{-1}$ corresponds to $\sim 3.5 \mathrm{pn} \mathrm{count} \mathrm{s}^{-1}$ (see text). The upper panels show the hardness ratio (counts between $2-10 \mathrm{keV}$ divided by those between $0.5-2 \mathrm{keV}$ ) with a $200 \mathrm{~s}$ binning.

rate reached its maximum of $\sim 4 \mathrm{~s}^{-1}$. The count rate then decreased by a factor $\sim 10$ and remained around $0.4 \mathrm{~s}^{-1}$ for the rest of the observation. In general, changes in intensity were accompanied by changes in the hardness ratio. IGR J17544-2619 was much brighter during the September 17 observation with the underlying MOS1 count rate decreasing from $\sim 2 \mathrm{~s}^{-1}$ to $\sim 0.5 \mathrm{~s}^{-1}$ with a number of flares up to $\sim 7.5 \mathrm{~s}^{-1}$ superposed (see Fig. 3). The peak MOS 1 count rate of $\sim 7.5 \mathrm{~s}^{-1}$ corresponds to $\sim 25 \mathrm{pn} \mathrm{s}^{-1}$, approximately a factor 6 higher than during the September 11 observation.

Power-density spectra of the September data reveal powerlaw shaped noise components, consistent with flaring on various time scales, with no obvious signatures such as pulsations or quasi-periodic oscillations.

\subsection{X-ray spectra}

All extracted spectra were rebinned to oversample the full width half maximum of the energy resolution by a factor 3 and to have additionally a minimum of 20 counts per bin to allow use of the $\chi^{2}$ statistic. In order to account for calibration uncertainties a $2 \%$ error was added quadratically to each spectral bin. Spectral uncertainties are given at $90 \%$ confidence.

Initially, simple absorbed spectral models were fit to the 0.5-10 keV September pn spectra integrated over each observation. For the September 11 spectrum, a power-law with $\alpha=2.25 \pm 0.15$ and $N_{\mathrm{H}}=(3.8 \pm 0.4) \times 10^{22}$ atom cm $^{-2}$ gives a $\chi^{2}$ of 151.9 for 142 degrees of freedom (d.o.f.), a thermal bremsstrahlung with $k T=5.5 \pm 0.9 \mathrm{keV}$ and 


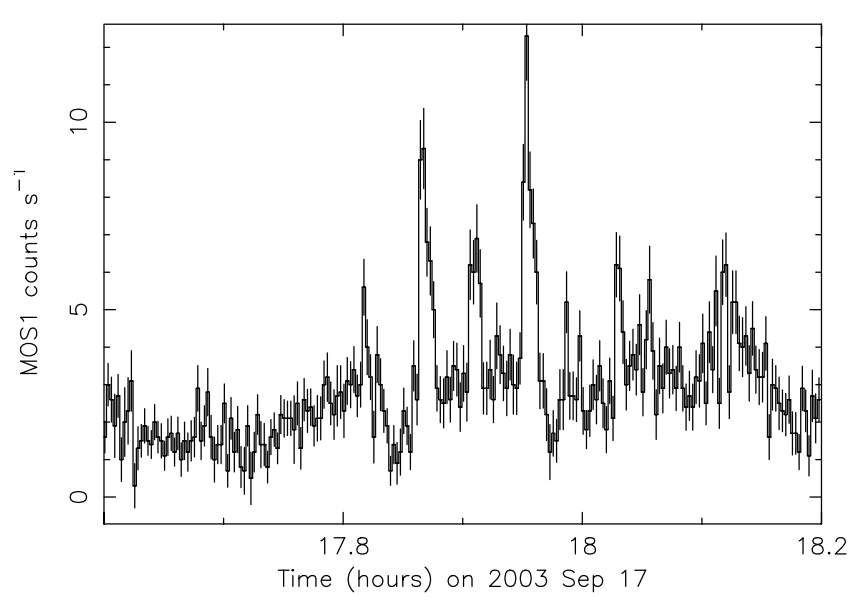

Fig. 3. Enlarged view of the $2-10 \mathrm{keV}$ September 17 light curve showing a number of short flares that took place between 17:45 and 18:15 UTC (binning is $10 \mathrm{~s}$ ).

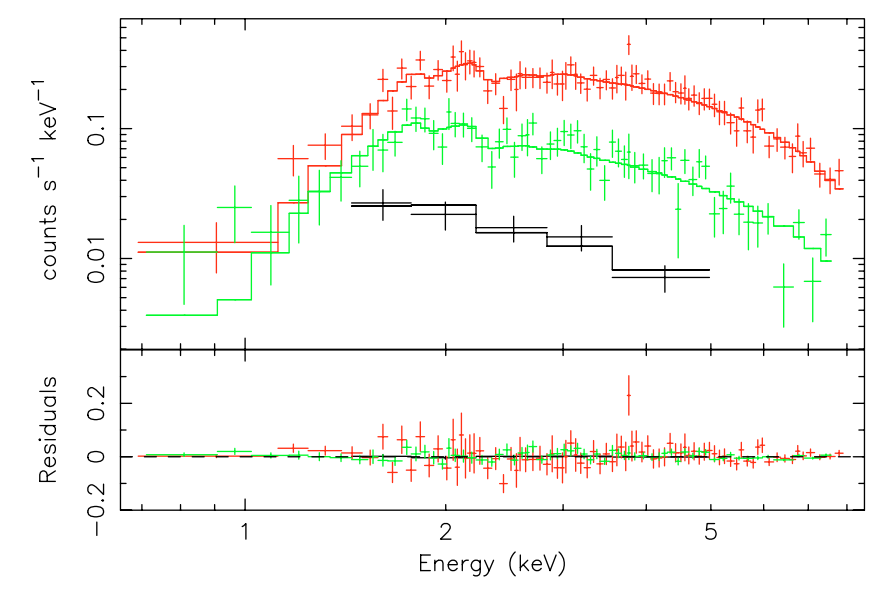

Fig. 4. Time selected (see text) pn count spectra from the September 11, 2003 observation. From bottom to top the 3 spectra correspond to pre-flare, post-flare and flaring intervals. The best-fit absorbed power-law models with the same value of $\alpha$ and different values of the normalisation and $N_{\mathrm{H}}$ for each spectrum are shown as histograms.

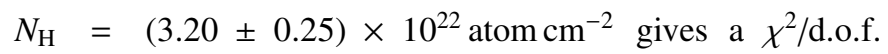
of 143.1/142 and blackbody with $k T=1.17 \pm 0.05 \mathrm{keV}$ and $N_{\mathrm{H}}=(1.48 \pm 0.20) \times 10^{22}$ atom $\mathrm{cm}^{-2}$ gives a $\chi^{2} /$ d.o.f. of 141.0/142. Since all the models describe the spectra with comparable goodness of fit, we cannot reliably distinguish between these simple spectral models. We therefore use the results from the power-law fit to estimate a mean $0.5-10 \mathrm{keV}$ unabsorbed flux of $1.5 \times 10^{-11} \mathrm{erg} \mathrm{cm}^{-2} \mathrm{~s}^{-1}$. The September 17 spectrum can be represented by a power-law model with $\alpha=$ $1.42 \pm 0.09$ and $N_{\mathrm{H}}=(2.50 \pm 0.17) \times 10^{22}$ atom cm $^{-2}$ to give a $\chi^{2} /$ d.o.f. of $283.8 / 215$. The mean $0.5-10 \mathrm{keV}$ unabsorbed flux is $7.5 \times 10^{-11} \mathrm{erg} \mathrm{cm}^{-2} \mathrm{~s}^{-1}$. Examination of the residuals indicates that the spectrum is not well fit $\lesssim 1 \mathrm{keV}$, which may indicate that there were variations in $N_{\mathrm{H}}$. This spectrum is significantly harder than that of September 11.

Next, the spectral changes during the September 11, 2003 observation were investigated. Three pn spectra were extracted corresponding to the pre-flare (until

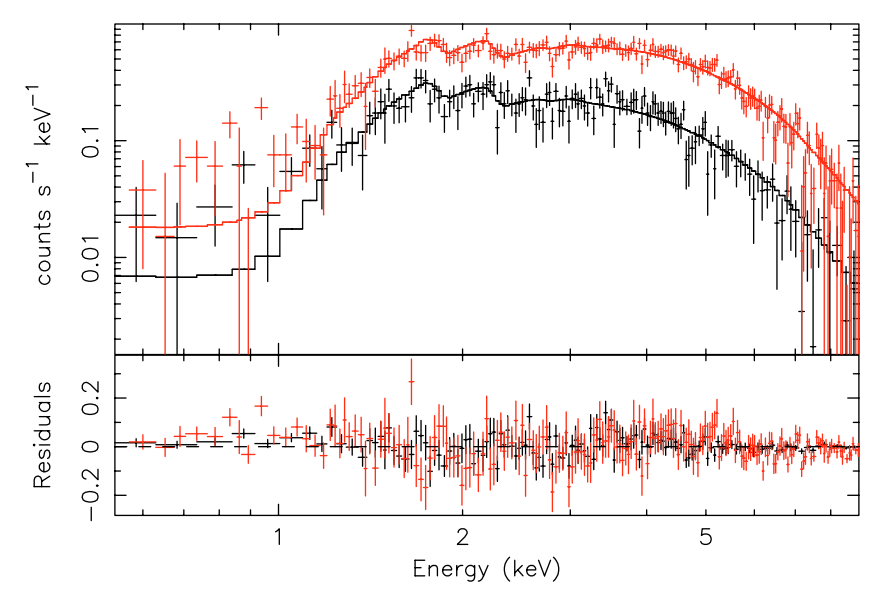

Fig. 5. Time selected MOS1 count spectra from the September 17, 2003 observation. The upper spectrum is from intervals when the $0.5-10 \mathrm{keV}$ count rate was $>2.5 \mathrm{~s}^{-1}$, the lower one from intervals below this value. The best-fit absorbed powerlaw models with the same value of $\alpha$ and different values of the normalisation and $N_{\mathrm{H}}$ for each spectrum are shown as histograms.

September 11, 2003 20:02 UTC), flare (September 11, 20:02 to 20:40 UTC), and post-flare (after September 11, 20:40 UTC) intervals. Background spectra were obtained simultaneously with the source spectra, since significant variations in the background count rate during the observation are present. The absorbed power-law model was used and the 3 spectra were fit simultaneously. First the value of $\alpha$ was constrained to be the same for all 3 spectra and the power-law normalisation, $k$, and $N_{\mathrm{H}}$ allowed to vary independently. This gives an acceptable fit with a $\chi^{2} /$ d.o.f. of $151.6 / 151$. The best-fit value of $\alpha$ is $2.20 \pm 0.09$ and $N_{\mathrm{H}}$ increases from $(1.9 \pm 0.5) \times 10^{22}$ atom $\mathrm{cm}^{-2}$ to $(4.3 \pm 0.2) \times 10^{22}$ atom cm$^{-2}$. The pre-flare $N_{\mathrm{H}}$ is consistent with the interstellar value in the direction of IGR J17544-2619 of $1.4 \times 10^{22} \mathrm{atom} \mathrm{cm}^{-2}$ (Dickey \& Lockman 1990). However, it is also possible to obtain a fit of similar quality if instead $\alpha$ is allowed to be different for the 3 spectra. Thus, it is not possible to draw any firm conclusions on the nature of the spectral changes during the observation, other than to say that they are consistent with either a changing $N_{\mathrm{H}}$ or $\alpha$.

To investigate whether similar variability was present during the September 17, 2003 observation the MOS1 data were subdivided in two intensity selected intervals above and below 2.5 count s$^{-1}$ and spectra extracted. MOS1 data were used due to the significantly longer exposure compared to the pn. The $0.5-10 \mathrm{keV}$ intensities were $3.9 \times 10^{-11} \mathrm{erg} \mathrm{cm}^{-2} \mathrm{~s}^{-1}$ and $1.1 \times 10^{-10} \mathrm{erg} \mathrm{cm}^{-2} \mathrm{~s}^{-1}$. The spectra were again fit together allowing either $\alpha$ or $N_{\mathrm{H}}$ to vary (together with the normalisation). Again, it is not possible to draw any firm conclusions since the variations are consistent with both a changing $N_{\mathrm{H}}$ or $\alpha$. With $\alpha$ constrained to be the same, the best-fit value of $\alpha$ is $1.43 \pm 0.09$ and $N_{\mathrm{H}}$ varies from $(1.97 \pm 0.15) \times 10^{22}$ atom cm $^{-2}$ to $(2.73 \pm 0.19) \times 10^{22}$ atom $\mathrm{cm}^{-2}$ for a $\chi^{2} /$ d.o.f. of $430.3 / 347$. We note that the fit to the high-intensity spectrum is worse than to the low-intensity spectrum. This may be caused by larger 
Table 2. Summary of spectral fit parameters for the September 2003 observations.

\begin{tabular}{lcccc}
\hline \hline Spectrum & $\begin{array}{c}N_{\mathrm{H}} \\
\left(10^{22} \text { atom cm } \mathrm{cm}^{-2}\right)\end{array}$ & $\alpha$ & $\chi^{2} /$ d.o.f. & $\begin{array}{c}0.5-10 \mathrm{keV} \text { flux } \\
\left(\mathrm{erg} \mathrm{cm}^{-2} \mathrm{~s}^{-1}\right)\end{array}$ \\
\hline Sep. 11 (pre flare) & $1.9 \pm 0.5$ & & & $8.8 \times 10^{-13}$ \\
Sep. 11 (flare) & $4.3 \pm 0.2$ & $2.20 \pm 0.09$ & $151.6 / 151$ & $4.0 \times 10^{-11}$ \\
Sep. 11 (post flare) & $3.1 \pm 0.3$ & & & $9.7 \times 10^{-12}$ \\
& & & & \\
Sep. 11 (all) & $3.8 \pm 0.4$ & $2.25 \pm 0.15$ & $151.9 / 142$ & $1.5 \times 10^{-11}$ \\
& & & & \\
Sep. 17 (high) & $2.0 \pm 0.2$ & $1.43 \pm 0.09$ & $430.3 / 347$ & $1.1 \times 10^{-10}$ \\
Sep. 17 (low) & $2.7 \pm 0.2$ & & & \\
& & & & \\
Sep. 17 (all) & $2.5 \pm 0.2$ & $1.42 \pm 0.09$ & $283.8 / 215$ & $7.5 \times 10^{-11}$ \\
\hline
\end{tabular}

spectral variations at high count rate. A summary of the fits to the different spectra is given in Table 2 .

\section{Discussion}

The mean $0.5-10 \mathrm{keV}$ unabsorbed luminosity of IGR J17544-2619 during the September 11 and 17, 2003 observations is $1.1 \times 10^{35} \mathrm{erg} \mathrm{s}^{-1}$ and $5.7 \times 10^{35} \mathrm{erg} \mathrm{s}^{-1}$ for an (assumed) distance of $8 \mathrm{kpc}$. The peak reached during the flare on September 17, 2003 is about $8.5 \times 10^{35} \mathrm{erg} \mathrm{s}^{-1}$. Such luminosities are only reached in systems where the compact object is a neutron star or black hole. The state observed in March 2003 corresponds to an $0.5-10 \mathrm{keV}$ luminosity of $<4 \times 10^{32} \mathrm{erg} \mathrm{s}^{-1}$. This upper limit is consistent with the luminosities observed for quiescent X-ray binary transients containing either a neutron star (e.g. Campana \& Stella 2003) or a black hole (e.g. Tomsick et al. 2003). The total dynamic flux range (quiescence to peak flaring) seen during the three XMM-Newton observations is a factor $\gtrsim 2000$. This is also typical for such transient X-ray binaries.

The (maximum) observed fluxes from the INTEGRAL observations (Sunyaev et al. 2003) may constrain the spectral model in the soft and hard X-ray bands, as well as the level of X-ray activity. Given the quoted fluxes, and assuming that the hard X-ray spectrum consists of a single power-law, the photon index was $\sim 4$ during the INTEGRAL observations. Assuming the interstellar $N_{\mathrm{H}}$, this would give an extrapolated absorbed $0.5-10 \mathrm{keV}$ flux of $\sim 6.4 \mathrm{Crab}$. If during the INTEGRAL observations the spectral index was $\sim 2.2$, as measured in the $0.5-10 \mathrm{keV}$ energy range on September 11, 2003, a high-energy cut-off at $\sim 14 \mathrm{keV}$ is required in the spectrum in order to explain the (maximum) $18-25 \mathrm{keV}$ and $25-60 \mathrm{keV}$ fluxes and the 50-100 keV flux upper limit. Extrapolating this spectrum gives an absorbed flux of $\sim 0.45 \mathrm{Crab}(0.5-10 \mathrm{keV})$. Note that this value would correspond to an unabsorbed luminosity of about $3 \times 10^{38} \mathrm{erg} \mathrm{s}^{-1}$ (at $8 \mathrm{kpc}$ ), similar to that reached by classical X-ray binary transients (e.g. Chen et al. 1997). Unfortunately, there are no closeby (i.e.- within hours) $R X T E$ All-Sky Monitor (ASM) measurements of IGR J17544-2619
(R. Remillard, private communication), to verify whether the source was active around the time of the INTEGRAL observations. The RXTE/ASM measurements within days of the INTEGRAL detections give typical upper limits of $\sim 50 \mathrm{mCrab}$. But since the source is highly variable in both soft and hard $\mathrm{X}$-rays, these do not provide stringent constraints either.

We note that the quiescent state and the low-level flaring seen with XMM-Newton and the high-level activity seen by INTEGRAL is reminiscent of SAX J1819.3-2525 (V4641 Sgr). This system also shows low-level activity around $10^{36} \mathrm{erg} \mathrm{s}^{-1}$ (in 't Zand et al. 2000), strong and short high-level activity (e.g. Revnivtsev et al. 2002), while in quiescence it reaches $\simeq 4 \times$ $10^{31} \mathrm{erg} \mathrm{s}^{-1}(0.3-8 \mathrm{keV}$; Tomsick et al. 2003). The compact object in SAX J1819.3-2525 is most probably a black hole (Orosz et al. 2001).

Our observed OM magnitudes, combined with the optical/infrared magnitudes reported by Rodriguez (2003), and assuming an absorption of $2 \times 10^{22}$ atom $\mathrm{cm}^{-2}$ and a distance of $8 \mathrm{kpc}$, are consistent with an early O-type companion. However, a foreground object cannot be ruled out; we note the possible presence of fainter optical candidates in the XMM-Newton error circle (Rodriguez 2003).

Future observations will hopefully shed more light on IGR J17544-2619. In particular, monitoring campaigns by INTEGRAL and multi-wavelength observations may allow the nature of the compact object to be elucidated.

Acknowledgements. Based on observations obtained with XMM-Newton, an ESA science mission with instruments and contributions directly funded by ESA member states and the USA (NASA).

\section{References}

Campana, S., \& Stella, L. 2003, in Proc. of the II BeppoSAX Meeting: The Restless High-Energy Universe, ed. E. P. J. van den Heuvel, J. J. M. in 't Zand, \& R. A. M. J. Wijers, Nucl. Phys. B. Suppl. Ser., Elsevier [arXiv: astro-ph/0309811]

Chen, W., Shrader, C. R., \& Livio, M. 1997, ApJ, 491, 312

den Herder, J. W., Brinkmann, A. C., Kahn, S. M., et al. 2001, A\&A, $365, \mathrm{~L} 7$ 
Dickey, J. M., \& Lockman, F. J. 1990, ARA\&A, 28, 215

González-Riestra, R., Santos-Lleó, M., \& Pérez-Martínez, R. 2003, IAU Circ., 8202

Grebenev, S. A., \& Lutovinov, A. A. 2003, ATel, 192

in 't Zand, J. J. M., Kuulkers, E., Bazzano, A., et al. 2001, A\&A, 357, 520

Jansen, F., Lumb, D., Altieri, B., et al. 2001, A\&A, 365, L1

Lebrun, F., Leray, J. P., Lavocat, P., et al. 2003, A\&A, 411, L141

Mason, K. O., Breeveld, A., Much, R., et al. 2001, A\&A, 365, L36

Matt, G., \& Guainazzi, M. 2003, MNRAS, 341, L13

Orosz, J. A., Kuulkers, E., van der Klis, M., et al. 2001, ApJ, 555, 489

Patel, S. K., Kouveliotou, C., Tennant, A., et al. 2003, ApJ, submitted

Revnivtsev, M., Gilfanov, M., Churazov, E., \& Sunyaev, R. 2002, A\&A, 391, 1013
Revnivtsev, M., Sazonov, S., Gilfanov, M., \& Sunyaev, R. 2003, AstL, 29, 587

Rodriguez, J. 2003, ATel, 194

Rodriguez, J., Tomsick, J. A., Forschini, L., et al. 2003, A\&A, 407, L41

Strüder, L., Briel, U., Dennerl, K., et al. 2001, A\&A, 365, L18

Sunyaev, R., Grebenev, S. A., Lutovinov, A. A., et al. 2003, ATel, 190

Tomsick, J. A., Corbel, S., Fender, R., et al. 2003, ApJ, 597, L133

Turner, M. J. L., Abbey, A., Arnaud, M., et al. 2001, A\&A, 365, L27

Walter, R., Rodriguez, J., Foschini, L., et al. 2003, A\&A, 411, L427

Wijnands, R. 2003, ATel, 191

Winkler, C., Courvoisier, T. J.-L., Di Cocco, G., et al. 2003, A\&A, 411, L1 